\title{
Distribution line models for loss calculation in three-phase three-wire power flow algorithms
}

DOI:

10.1109/TDC.2004.1432372

Link to publication record in Manchester Research Explorer

\section{Citation for published version (APA):}

Ochoa, L. F., \& Padilha-Feltrin, A. (2004). Distribution line models for loss calculation in three-phase three-wire power flow algorithms. In IEEE/PES Transmission \& Distribution Latin America 2004 (pp. 173-178)

https://doi.org/10.1109/TDC.2004.1432372

\section{Published in:}

IEEE/PES Transmission \& Distribution Latin America 2004

\section{Citing this paper}

Please note that where the full-text provided on Manchester Research Explorer is the Author Accepted Manuscript or Proof version this may differ from the final Published version. If citing, it is advised that you check and use the publisher's definitive version.

\section{General rights}

Copyright and moral rights for the publications made accessible in the Research Explorer are retained by the authors and/or other copyright owners and it is a condition of accessing publications that users recognise and abide by the legal requirements associated with these rights.

\section{Takedown policy}

If you believe that this document breaches copyright please refer to the University of Manchester's Takedown Procedures [http://man.ac.uk/04Y6Bo] or contact uml.scholarlycommunications@manchester.ac.uk providing relevant details, so we can investigate your claim.

\section{OPEN ACCESS}




\title{
Distribution Line Models Analysis for Loss Calculation within Three-phase Three-wire Power Flow Algorithms
}

\author{
L. F. Ochoa, Student Member, IEEE, and A. Padilha-Feltrin, Member, IEEE
}

\begin{abstract}
Three-phase three-wire power flow algorithms, as any tool for power systems analysis, require reliable impedances and models in order to obtain accurate results. Kron's reduction procedure, which embeds neutral wire influence into phase wires, has shown good results when three-phase three-wire power flow algorithms based on current summation method were used. However, Kron's reduction can harm reliabilities of some algorithms whose iterative processes need loss calculation (power summation method). In this work, three three-phase three-wire power flow algorithms based on power summation method, will be compared with a three-phase four-wire approach based on backward-forward technique and current summation. Two fourwire unbalanced medium-voltage distribution networks will be analyzed and results will be presented and discussed.
\end{abstract}

Index Terms-- Distribution networks, losses, unbalance, threephase power flow, distribution line models.

\section{INTRODUCTION}

$\mathrm{P}$ OWER flow is a very important and fundamental tool for analysis of any power system. Several efficient power flow solution techniques for radial or weakly meshed distribution networks have been developed over the last few decades. Moreover, in order to consider the inherently unsymmetrical line segments and typically unbalanced loads, power flow algorithms had to be expanded to consider three-phase threewire systems or even three-phase four-wire systems, with explicit neutral wire. However, it must be understood that the results of the power-flow studies will only be as reliable as the impedances and models that will be used.

Some three-phase power flow algorithms are based on the power loss calculation within the iterative process in order to consider the total power demand [1], [2], [3] (power summation method). In these algorithms, when neutral wire is merged into phase wires using Kron's reduction for obtaining impedances, loss calculation, computed as the phase resistance times the current squared $\left(I^{2} R\right)$, could harm their reliability affecting final values of voltages and currents.

In this work, reliability of three three-phase three-wire power flow algorithms based on power summation method,

The first author would like to thank CNPq (process no. 141600/03-9), for the financial support.

L. F. Ochoa and A. Padilha-Feltrin are with Universidade Estadual Paulista (UNESP) Ilha Solteira, SP, Brazil.

e-mail: luis_ochoa@ieee.org, padilha@dee.feis.unesp.br will be compared with a three-phase four-wire approach based on backward-forward technique and current summation (avoiding loss calculation within the algorithm) [4]. A special focus on the reduced impedances influence will be given. Two four-wire unbalanced medium-voltage distribution networks will be analyzed and results will be presented and discussed.

\section{THREE-PHASE THREE-WIRE POWER FlOW AlgORITHMS}

Single-phase power flow algorithms from [1] (Cespedes), [2] (Baran and $\mathrm{Wu}$ ) and [3] (Luo and Semlyen) for balanced distribution networks are extended for an unbalanced scenario where computations of phase state variables are needed.

In this work, each line to be used in the following threephase three-wire power flow algorithms will be represented by the following $3 \times 3$ matrix:

$$
\left[Z_{l}\right]_{3 \times 3}=\left[\begin{array}{ccc}
\bar{Z} a a_{l} & \bar{Z} a b_{l} & \bar{Z} a c_{l} \\
\bar{Z} a b_{l} & \bar{Z} b b_{l} & \bar{Z} b c_{l} \\
\bar{Z} a c_{l} & \bar{Z} b c_{l} & \bar{Z} c c_{l}
\end{array}\right] \quad \begin{aligned}
& \text { where } \\
& a, b, c-\text { phase lines }
\end{aligned}
$$

\section{A. Cespedes' Power Flow Algorithm}

The basic formula for single-phase solution is used for the three phases. Considering Fig. 1, the following equations will be applied for the forward sweep in the Cespedes' three-phase three-wire power flow algorithm.

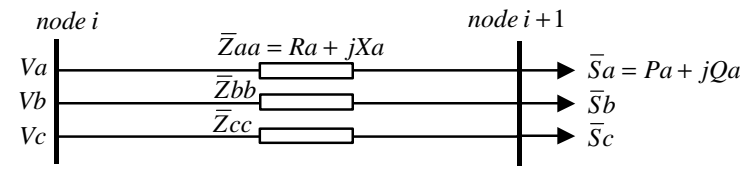

Fig. 1. Three-phase three-wire section for Cespedes' algorithm.

$$
\begin{aligned}
& V a_{i+1}^{4}+\left(2 \cdot\left(P a_{i+1} R a+Q a_{i+1} X a\right)-V a_{i}^{2}\right) \cdot V a_{i+1}^{2}+\left|\bar{S} a_{i+1}\right|^{2} \cdot\left|\bar{Z} a a_{i+1}\right|^{2}=0 \\
& V b_{i+1}^{4}+\left(2 \cdot\left(P b_{i+1} R b+Q b_{i+1} X b\right)-V b_{i}^{2}\right) \cdot V b_{i+1}^{2}+\left|\bar{S} b_{i+1}\right|^{2} \cdot\left|\bar{Z} b b_{i+1}\right|^{2}=0 \\
& V c_{i+1}^{4}+\left(2 \cdot\left(P c_{i+1} R c+Q c_{i+1} X c\right)-V c_{i}^{2}\right) \cdot V c_{i+1}^{2}+\left|\bar{S} c_{i+1}\right|^{2} \cdot\left|\bar{Z} c c_{i+1}\right|^{2}=0
\end{aligned}
$$

The above equations do not depend on the phase angle and have straightforward solutions. Equivalent power at node $i+1$ is calculated by summing all loads of the network fed through the node, including losses.

The solution method has the following steps:

1. Assume a voltage magnitude for the initial load 
estimation.

2. Calculate the equivalent power for each node. This is the backward sweep, from end nodes to source nodes.

3. Starting from the source(s) node(s) and using (1), (2) and (3), calculate the equivalent power voltage for all nodes. This is the forward sweep, from source nodes to end nodes.

4. With the new voltages, recalculate the losses. If the total losses variation with respect to the previous iteration is greater than a specified error, go to step 2.

Since this work is aimed at maintaining Cespedes' original equations, they cannot be directly adapted for considering matrix 3x3 mutual impedances influences. Therefore, only self impedances are considered in the presented formulation. A way to insert mutual impedances influences is the method proposed in [7].

\section{B. Baran-Wu's Power Flow Algorithm}

Considering Fig. 2, where the power supplied by the substation and root nodes of laterals and sublaterals are assumed to be known, three-phase power flow in a radial distribution network can be described by the following recursive equations:

$$
\begin{gathered}
\bar{S} a_{k+1}=\bar{S} a_{k}-\bar{Z} a a_{k+1} \frac{\left|\bar{S} a_{k}\right|^{2}}{V a_{k}^{2}}-\bar{S} a_{L k+1} \\
V a_{k+1}^{2}=V a_{k}^{2}-2 \cdot\left(R a_{k+1} P a_{k}+X a_{k+1} Q a_{k}\right)+\left|\bar{Z} a a_{k+1}\right|^{2} \frac{\left|\bar{S} a_{k}\right|^{2}}{V a_{k}^{2}} \\
\bar{S} b_{k+1}=\bar{S} b_{k}-\bar{Z} b b_{k+1} \frac{\left|\bar{S} b_{k}\right|^{2}}{V b_{k}^{2}}-\bar{S} b_{L k+1} \\
V b_{k+1}^{2}=V b_{k}^{2}-2 \cdot\left(R b_{k+1} P b_{k}+X b_{k+1} Q b_{k}\right)+\left|\bar{Z} b b_{k+1}\right|^{2} \frac{\left|\bar{S} b_{k}\right|^{2}}{V b_{k}^{2}} \\
\bar{S} c_{k+1}=\bar{S} c_{k}-\bar{Z} c c_{k+1} \frac{\left|\bar{S} c_{k}\right|^{2}}{V c_{k}^{2}}-\bar{S} c_{L k+1} \\
V c_{k+1}^{2}=V c_{k}^{2}-2 \cdot\left(R c_{k+1} P c_{k}+X c_{k+1} Q c_{k}\right)+\left|\bar{Z} c c_{k+1}\right|^{2} \frac{\left|\bar{S} c_{k}\right|^{2}}{V c_{k}^{2}}
\end{gathered}
$$

Where

$$
\begin{aligned}
& \bar{S} a_{k}, \bar{S} b_{k}, \bar{S} c_{k} \quad: \text { complex power flows for phases } a, b \\
& V a_{k}, V b_{k}, V c_{k} \quad: \quad \text { bus voltage magnitudes for phases } a, b \\
& \bar{Z} a a_{k+1} \quad: \text { self impedance in phase } a \text { of branch } k+1 \text {. } \\
& \bar{Z} a b_{k+1} \quad: \text { mutual impedance between phases } a \text { and } \\
& b \text { of branch } k+1 \text {. }
\end{aligned}
$$

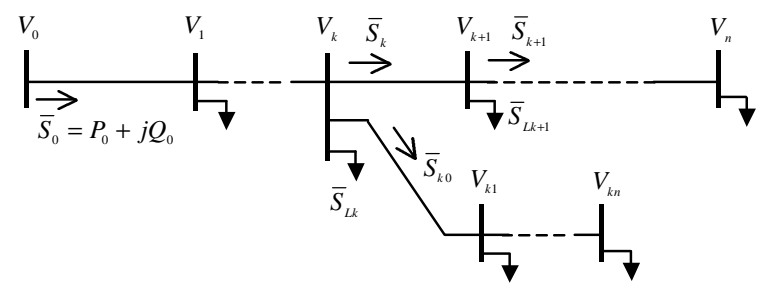

Fig. 2. One line diagram of a section for Baran-Wu's algorithm.

In Fig. 2, $V_{0}$ represents the substation bus voltage magnitude that is assumed to be constant. Terminal condition for the iterative process is having leaving complex powers at end nodes smaller than a specified error.

The solution method has the following steps:

1. Calculate the equivalent power (sum of fed loads) for each root node (substation and initial nodes of laterals and sublaterals).

2. Assume a voltage magnitude for the substation node.

3. Solve power flow in the main feeder by using (4), (5) and (6). Solve power flow in laterals and sublaterals considering the voltages magnitudes obtained in the previous calculation (e.g. main feeder).

4. If the leaving end-nodes complex powers are greater than a specified error, add the total losses of the analyzed set of branches to the equivalent powers of each root node and go to step 3 .

Since this work is aimed at maintaining Baran-Wu's original voltage equations and power equations (4), (5) and (6), they cannot be directly adapted for considering matrix $3 \times 3$ mutual impedances influences. A way to insert mutual impedances influences is the method proposed in [7].

\section{Luo-Semlyen's Power Flow Algorithm}

This algorithm considers backward and forward sweeps for computing complex powers and voltages, respectively. The backward sweep serves to sum the load powers and the power losses from the end nodes to the root. The forward sweep establishes the nodal voltages from the root to the end nodes based on the power flows obtained in the backward sweep.

In Fig. 3, phase complex powers with index $i+1$ are the sum of loads in the downstream nodes (including node $i+1$ ) and losses in the downstream branches (calculated with an specified initial voltage).

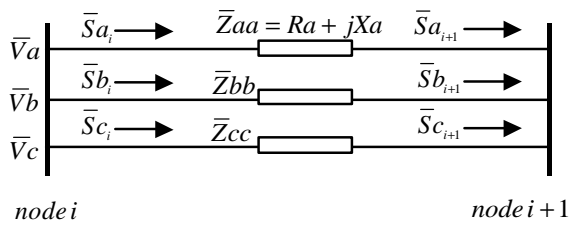

Fig. 3. Three-phase three-wire section for Luo-Semlyen's algorithm.

In the backward sweep, the upstream node phase complex powers (node $i$ ) are calculated from:

$$
\begin{aligned}
& \bar{S} a_{i}=\bar{S} a_{i+1}+\bar{Z} a a \cdot \frac{\left|\bar{S} a_{i+1}\right|^{2}}{\left|\bar{V} a_{i+1}\right|^{2}} \\
& \bar{S} b_{i}=\bar{S} b_{i+1}+\bar{Z} b b \cdot \frac{\left|\bar{S} b_{i+1}\right|^{2}}{\left|\bar{V} b_{i+1}\right|^{2}} \\
& \bar{S} c_{i}=\bar{S} c_{i+1}+\bar{Z} c c \cdot \frac{\left|\bar{S} c_{i+1}\right|^{2}}{\left|\bar{V} c_{i+1}\right|^{2}}
\end{aligned}
$$

In the forward sweep, with the upstream node phase voltages known (node $i$ ), the phase voltages in node $i+1$ are 
computed from:

$$
\begin{aligned}
& \bar{V} a_{i+1}=\bar{V} a_{i}-\bar{Z} a a \cdot\left(\frac{\bar{S} a_{i}}{\bar{V} a_{i}}\right)^{*}-\bar{Z} a b \cdot\left(\frac{\bar{S} b_{i}}{\bar{V} b_{i}}\right)^{*}-\bar{Z} a c \cdot\left(\frac{\bar{S} c_{i}}{\bar{V} c_{i}}\right)^{*} \\
& \bar{V} b_{i+1}=\bar{V} b_{i}-\bar{Z} b b \cdot\left(\frac{\bar{S} b_{i}}{\bar{V} b_{i}}\right)^{*}-\bar{Z} a b \cdot\left(\frac{\bar{S} a_{i}}{\bar{V} a_{i}}\right)^{*}-\bar{Z} b c \cdot\left(\frac{\bar{S} c_{i}}{\bar{V} c_{i}}\right)^{*} \\
& \bar{V} c_{i+1}=\bar{V} c_{i}-\bar{Z} c c \cdot\left(\frac{\bar{S} c_{i}}{\bar{V} c_{i}}\right)^{*}-\bar{Z} a c \cdot\left(\frac{\bar{S} a_{i}}{\bar{V} a_{i}}\right)^{*}-\bar{Z} b c \cdot\left(\frac{\bar{S} b_{i}}{\bar{V} b_{i}}\right)^{*}
\end{aligned}
$$

In this extended approach of Luo-Semlyen's algorithm nodal voltages are considered as complex variables, and influence of mutual impedances is inserted in the voltage drop calculation.

After performing the backward and forward sweeps computation total losses are calculated. If total losses variation with respect to the previous iteration is greater than a specified error, another backward-forward process has to be performed.

\section{THREE-PHASE FouR-WIRE POWER FLOW ALGORITHM}

In order to compare results obtained with above power flow algorithms, the backward-forward based three-phase four-wire power flow algorithm, which uses $5 \times 5$ network representation, described in [4] was adopted.

Since ground currents analysis is not part of this work focus, for this algorithm each line will be represented by a $4 \times 4$ matrix:

$$
\left[Z_{l}\right]_{4 \times 4}=\left[\begin{array}{llll}
\bar{Z}_{a a} & \bar{Z}_{a b} & \bar{Z}_{a c} & \bar{Z}_{a n} \\
\bar{Z}_{a b} & \bar{Z}_{b b} & \bar{Z}_{b c} & \bar{Z}_{b n} \\
\bar{Z}_{a c} & \bar{Z}_{b c} & \bar{Z}_{c c} & \bar{Z}_{c n} \\
\bar{Z}_{a n} & \bar{Z}_{b n} & \bar{Z}_{c n} & \bar{Z}_{n n}
\end{array}\right] \begin{aligned}
& \text { where } \\
& a, b, c-\text { phase lines } \\
& n-\text { neutral wire }
\end{aligned}
$$

Fig. 3 shows the line model used for this power flow algorithm:

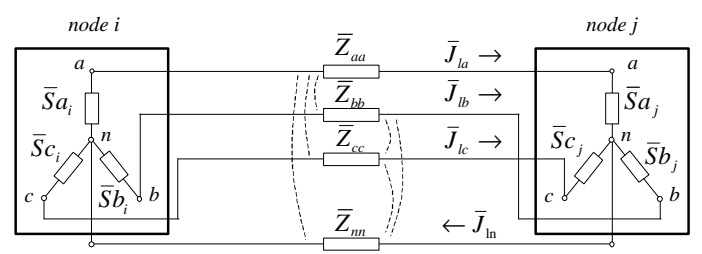

Fig. 4. Model of the three-phase four-wire isolated-neutral distribution line.

Assuming the root node to be the slack node with known voltage magnitude and angle, the $4 \times 4$ iterative algorithm to solve the radial system consists of three steps, is as follows.

At iteration $k$ :

1. Nodal current calculation

$$
\left[\begin{array}{c}
\bar{I} a_{i} \\
\bar{I} b_{i} \\
\bar{I} c_{i} \\
\bar{I} n_{i}
\end{array}\right]^{(k)}=\left[\begin{array}{c}
\left(\bar{S} a_{i} / \bar{V} a_{i}\right)^{(k-1)^{*}} \\
\left(\bar{S} b_{i} / \bar{V} b_{i}\right)^{(-1)^{*}} \\
\left(\bar{S} c_{i} / \bar{V} c_{i}\right)^{(-1)^{*}} \\
\left(\bar{I} a_{i}^{(k)}+\bar{I} b_{i}^{(k)}+\bar{I} c_{i}^{(k)}\right)
\end{array}\right]
$$

where

$$
\begin{aligned}
& \bar{I} a_{i}, \bar{I} b_{i}, \bar{I} c_{i} \quad: \quad \text { current injections for phases } a, b \text { and } c \text { at } \\
& \text { node } i \text {. } \\
& \bar{I} n_{i} \quad: \quad \text { current injection for neutral wire at node } i . \\
& \bar{S} a_{i}, \bar{S} b_{i}, \bar{S} c_{i}: \text { complex power injections (loads) for } \\
& \text { phases } a, b \text { and } c \text { at node } i \text {. }
\end{aligned}
$$

2. Backward sweep - section current calculation

Starting from the line section in the last layer and moving towards the root node, the current in line section $l$ is:

$$
\left[\begin{array}{l}
\bar{J} a_{l} \\
\bar{J} b_{l} \\
\bar{J} c_{l} \\
\bar{J} n_{l}
\end{array}\right]^{(k)}=-\left[\begin{array}{l}
\bar{I} a_{j} \\
\bar{I} b_{j} \\
\bar{I} c_{j} \\
\bar{I} n_{j}
\end{array}\right]^{(k)}+\sum_{m \in M}\left[\begin{array}{l}
\bar{J} a_{m} \\
\bar{J} b_{m} \\
\bar{J} c_{m} \\
\bar{J} n_{m}
\end{array}\right]^{(k)}
$$

where

$\bar{J} a_{l}, \bar{J} b_{l}, \bar{J} c_{l}:$ current flows for phases $a, b$ and $c$ on line section $l$.

$\bar{J} n_{l} \quad: \quad$ current flow for neutral wire on line section $l$.

M : set of line sections connected downstream to node $j$.

3. Forward sweep - nodal voltage calculation

Starting from the first layer and moving towards the last layer, the voltage at node $j$ is:

$$
\left[\begin{array}{l}
\bar{V} a_{j} \\
\bar{V} b_{j} \\
\bar{V} c_{j} \\
\bar{V} n_{j}
\end{array}\right]^{(k)}=\left[\begin{array}{l}
\bar{V} a_{i} \\
\bar{V} b_{i} \\
\bar{V} c_{i} \\
\bar{V} n_{i}
\end{array}\right]^{(k)}-\left[\begin{array}{llll}
\bar{Z}_{a a} & \bar{Z}_{a b} & \bar{Z}_{a c} & \bar{Z}_{a n} \\
\bar{Z}_{a b} & \bar{Z}_{b b} & \bar{Z}_{b c} & \bar{Z}_{b n} \\
\bar{Z}_{a c} & \bar{Z}_{b c} & \bar{Z}_{c c} & \bar{Z}_{c n} \\
\bar{Z}_{a n} & \bar{Z}_{b n} & \bar{Z}_{c n} & \bar{Z}_{n n}
\end{array}\right]\left[\begin{array}{l}
\bar{J} a_{l} \\
\bar{J} b_{l} \\
\bar{J} c_{l} \\
\bar{J} n_{l}
\end{array}\right]^{(k)}
$$

\section{Convergence criterion}

After these steps are executed in one iteration, the power mismatches at each node for all phases, neutral wire and ground are calculated.

If the real or imaginary part of any of the power mismatches is greater than a convergence criterion, steps 1,2 and 3 are repeated until convergence is achieved.

The initial voltage for all nodes should be equal to the root node voltage magnitude considering angular displacement for each phase. Neutral voltage at root node is equal to zero.

\section{TEST NETWORKS}

Described three-phase three-wire power flow algorithms will be applied on the following two medium voltage distribution networks:

MV-475: 475-bus real-life four-wire three-phase MV system from Assis city, SP-Brazil (data supplied by Grupo Rede). The MV-475 is mainly a rural network with total demand of $6248.79 \mathrm{~kW}$. Almost $75 \%$ of the loads are concentrated in a radius of $10 \mathrm{~km}$ from the root node (the most distant node is $16.5 \mathrm{~km}$ from the substation). Line-toline base voltage is $\mathrm{V}_{\mathrm{b}}=13.8 \mathrm{kV}$; and

IEEE-34: 34-bus IEEE four-wire three-phase MV feeder (Fig. 5) [5]. The total demand is $1770.0 \mathrm{~kW}$, and $72 \%$ of 
the loads are concentrated $56 \mathrm{~km}$ far away from the root node (the most distant node is $59 \mathrm{~km}$ from the substation). Line-to-line base voltage is $\mathrm{V}_{\mathrm{b}}=24.9 \mathrm{kV}$.

Simplifying, the autotransformer $24.9 / 4.16 \mathrm{kV} / \mathrm{kV}$ in the original IEEE-34 test feeder is replaced with the line and the network is modeled with the single voltage level. The automatic voltage regulator is also not represented.

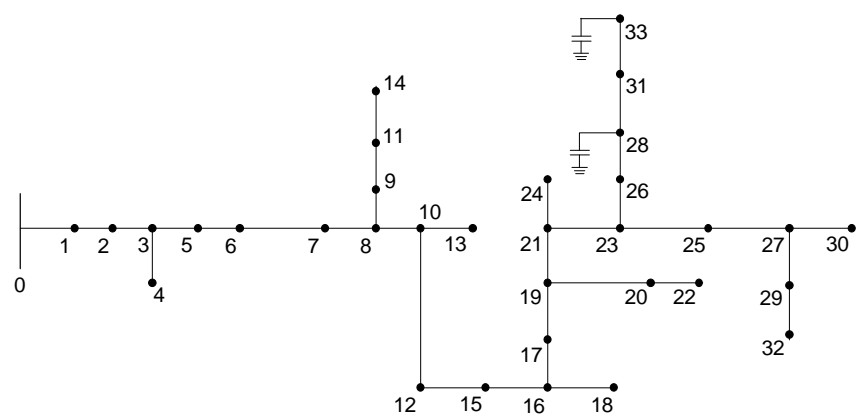

Fig. 5. IEEE-34 test feeder.

\section{APPLICATIONS}

Cespedes, Baran-Wu and Luo-Semlyen's three-phase threewire power flow algorithms will use the elements of $3 \times 3$ matrix line impedance produced by performing Kron's reduction from a $4 \times 4$ matrix which includes the neutral wire, i.e. matrix $\left[Z_{l}\right]_{3 \times 3}$ presented in section II is obtained by performing Kron's reduction from matrix $\left[Z_{l}\right]_{4 \times 4}$ presented in section III.

Three-phase four-wire power flow algorithm previously presented will use the original $4 \times 4$ matrix line impedance, considering neutral wire. Calculation of the $4 \times 4$ matrix line impedance will consider $100 \Omega$-m of ground resistivity [6].

Loads for the test networks IEEE-34 and MV-475 were modeled as constant power. Errors used for the convergence of the four algorithms were $0.5 \mathrm{~kW}$ and $0.5 \mathrm{kVAr}$.

Fig. 6 shows the voltage magnitudes of phase $c$ computed with the four described algorithms for MV-475 network. Selected path presented the greater voltage magnitude mismatches respect to the three-phase four-wire algorithm, since is the longer feeder in the network. Mismatches were greater in phase $c$, than in phases $a$ and $b$.

Results obtained for Cespedes and Baran-Wu's algorithms were almost the same. However, when comparing those results with the three-phase four-wire algorithm the greatest mismatch was $2.2 \%$. Luo-Semlyen's approach presented even greater difference with a maximum mismatch of $2.5 \%$.

Fig. 7 shows the voltage magnitudes of phase $b$ computed with the three described algorithms for IEEE-34 network. Selected path presented the greater voltage magnitude mismatches respect to the three-phase four-wire algorithm, mainly due to the load concentration and the long distance that raises the magnitude of the voltage drops. Mismatches were greater in phase $b$, than in phases $a$ and $c$.

Results obtained for Cespedes and Baran-Wu's algorithms were very similar. Nevertheless, results compared with those from the three-phase four-wire algorithm the greatest mismatch was $8.3 \%$. Luo-Semlyen's algorithm presented a better performance than the Cespedes and Baran-Wu's approaches with a maximum mismatch of $2.5 \%$.

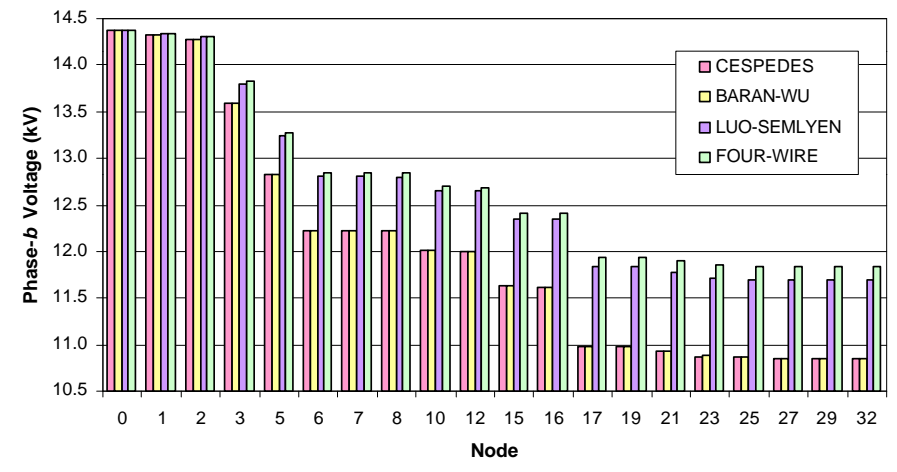

Fig. 7. Phase- $b$ voltage magnitude for IEEE-34 network.

The high mismatch of IEEE-34 network results compared with the MV-475 network is due to the inherent differences between both networks. As stated before, IEEE-34 has its main loads far away from the substation whereas MV- 475 has the major demand closer to the root node. This load characteristic, plus the network length, makes the IEEE-34 network produce more losses than MV-475. The greater the looses are, the greater the error of Cespedes and Baran-Wu's algorithms. Luo-Semlyen approach shows smaller errors due to the presence of mutual line impedance in the voltage calculation.

Both Cespedes and Baran-Wu extended algorithms, obtained very similar results. However, Cespedes' approach was faster, in both computational time and number of iterations, than Baran-Wu's approach. Moreover, Cespedes' approach was simpler for its computational implementation. In the other hand, Luo-Semlyen extended algorithm presented similar performance than Cespedes'.

Though the use of the reduced-matrix self impedances in both analyzed three-phase three-wire algorithms leads to incorrect results, original phase wires impedances could be used in order to computed results closer to the three-phase four-wire approach. 


\section{Accepted Paper}

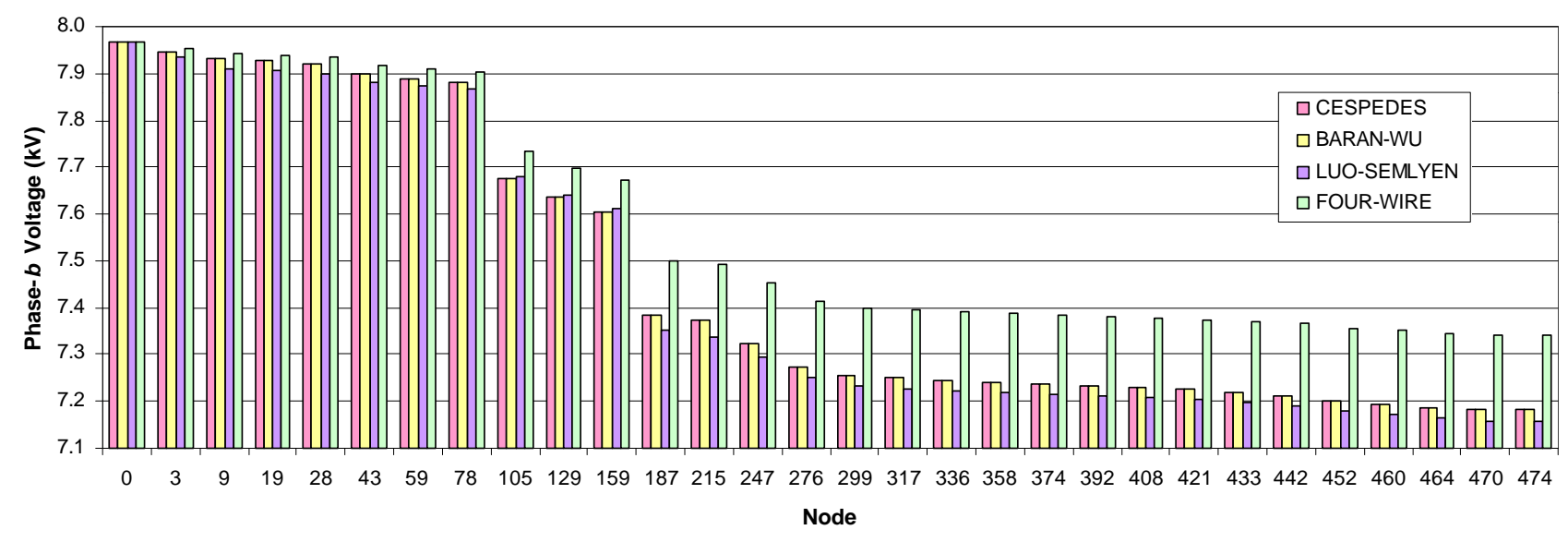

Fig. 6. Phase- $c$ voltage magnitude for MV-475 network, rural feeder.

Fig. 8 shows the new voltage magnitudes of phase $c$ computed by using original phase wires impedances in Cespedes, Baran-Wu and Luo-Semlyen's algorithms. Results obtained previously with the three-phase four-wire approach were maintained. In this case, mismatches diminished (the greatest mismatch was $0.9 \%$ for Cespedes and Baran-Wu's approaches and $2.0 \%$ for Luo-Semlyen's approach). This means that a significant improvement could be made with the results if original impedances (no embedded influence of neutral wire nor ground resistivity) of phase wires are used in analyzed three-phase three-wire power flow algorithms.

The same improvement was found when original phase wires impedances were used for IEEE-34 network (Fig. 9). The greatest mismatch was $2.9 \%$ for Cespedes and BaranWu's approaches and $0.9 \%$ for Luo-Semlyen's approach.

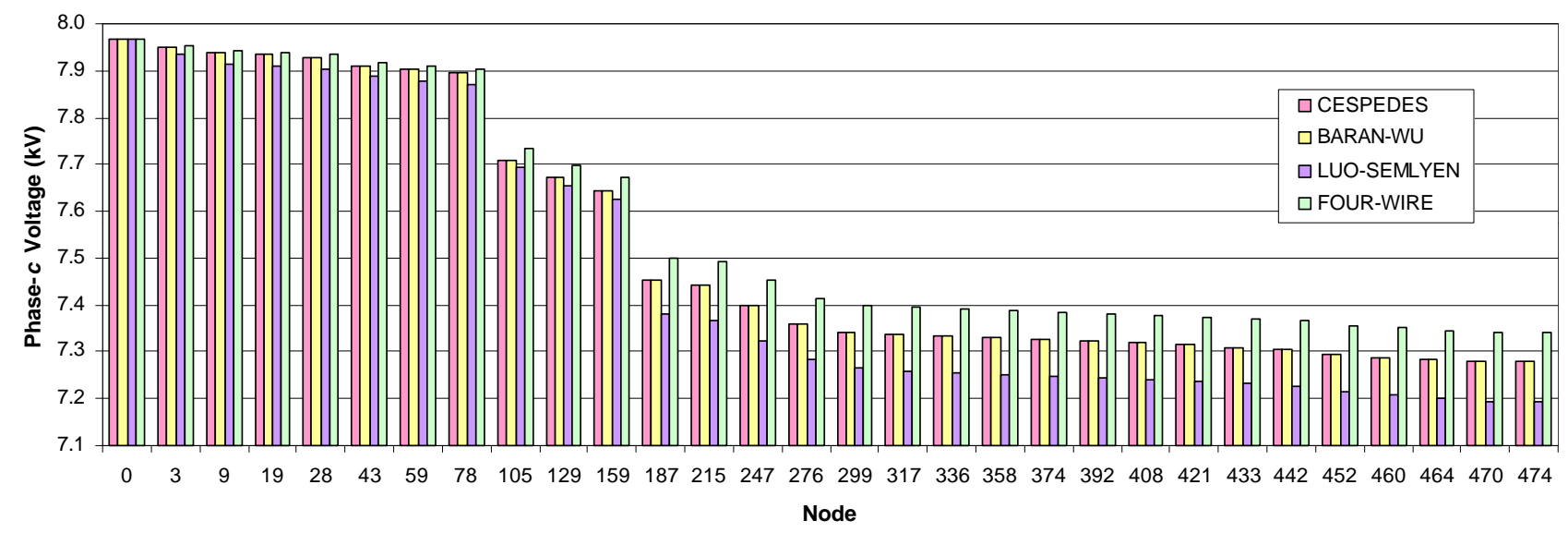

Fig. 8. Phase- $c$ voltage magnitude for MV-475 network, rural feeder (improved results).

As presented, both algorithms, Cespedes and Baran-Wu, could have their results noticeably improved, however due to the neglected influence of mutual impedances in those power flow approaches, closer results to the three-phase four-wire algorithm cannot be achieved. Though Luo-Semlyen's approach results were improved, it was not significant due to already consider line mutual impedances.

One can notice that Cespedes and Baran-Wu's approaches worked, each one, as three single-phase power flow algorithms since no mutual impedance was considered. Another manner to improve these three-phase three-wire "decoupled" algorithms is by applying the methodology proposed in [7], where effects of mutual impedances are modeled as branch voltage sources or additional bus injections.

Finally, it is important to recognize that three-phase threewire power flow algorithms are not able to directly compute neutral and ground voltages and currents. Those parameters, which are important in some distribution power systems applications, can be directly obtained by using the presented three-phase four-wire algorithm [4]. 


\section{Accepted Paper}

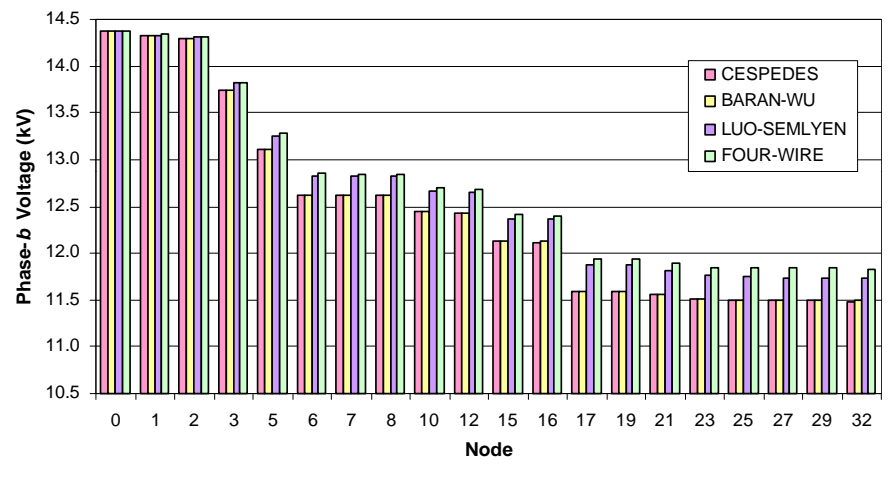

Fig. 9. Phase- $b$ voltage magnitude for IEEE-34 network (improved results).

\section{CONCLUSIONS}

In this paper it was presented a comparison between the three-phase four-wire power flow algorithm and Cespedes, Baran-Wu and Luo-Semlyen's extended algorithms (threephase three-wire) when the influence of neutral wire (Kron's reduction) and real ground (ground resistivity) are considered in the $3 \times 3$ line impedance matrix.

It was shown that the best manner to compute power flow while using those three-phase three-wire approaches, is to consider the $3 \times 3$ original matrix impedances, neglecting ground resistivity and neutral wire influences.

Both algorithms, Cespedes and Baran-Wu, obtained almost the same results, whereas Cespedes' approaches presented better performance in terms of convergence speed and computation time.

Errors obtained from the usage of line impedance matrices computed from Kron's reduction and considering ground resistivity in power summation based algorithms appear because of the internal loss calculation, which should consider the original wires impedances.

\section{REFERENCES}

[1] R. Cespedes, "New method for the analysis of distribution networks," IEEE Trans. On Power Systems, vol. 5, no. 1, pp. 391-396, Jan. 1990.

[2] M. E. Baran and F. F. Wu, "Network reconfiguration in distribution systems for loss reduction and load balancing," IEEE Trans. On Power Delivery, vol. 4, no. 2, pp. 1401-1407, April 1989.

[3] G. X. Luo, and A. Semlyen, "Efficient load flow for large weakly meshed networks," IEEE Trans. on Power Systems, vol. 5, no. 4, pp. 1309-1316, Nov. 1990.

[4] R. M. Ciric, A. Padilha, and L. F. Ochoa, "power flow in four-wire distribution networks-general approach," IEEE Trans. on Power Systems, vol. 18, no. 4, pp. 1283-1290, Nov. 2003.

[5] W. H. Kersting, "Radial distribution test feeders," IEEE Trans. on Power Systems, vol. 6, no. 3, pp. 975-985, Aug. 1991.

[6] J. R. Carson, "Wave propagation in overhead wires with ground return," Bell System Technical Journal, 1927.

[7] E. R. Ramos, A. G. Expósito, and G. A. Cordero, "Quasi-coupled threephase radial load flow," IEEE Trans. on Power Systems, vol. 19, no. 2, pp. 776-781, May 2004.

\section{BIOGRAPHIES}

Luis F. Ochoa (S'01) graduated from the National Engineering University (U.N.I.) - Lima, Peru, in 2000. He obtained the M.Sc. degree from UNESP Ilha Solteira, Brazil in 2003. Currently he is towards the Ph.D. at UNESP Ilha Solteira, Brazil. His research interests are distribution system analysis and distributed generation.
Antonio Padilha Feltrin (M'89) obtained the B.Sc. degree from EFEI, the M.Sc. and Ph.D. from UNICAMP, Brazil. He is currently an Associate Professor at UNESP - Ilha Solteira, SP, Brazil. From 1995 to 1997 he was a Visiting Faculty at ECE Department of University of Wisconsin - Madison. His main interests are in analysis and control of power systems. 